\title{
Segundo congreso internacional de la Sociedad Portuguesa de Medicina del Trabajo
}

\author{
Mari Cruz Rodríguez-Jareño ${ }^{1,2,3,4}$ \\ 'Societat Catalana de Salut Laboral, Barcelona, España \\ ${ }^{2}$ Sociedad Española de Medicina y Seguridad en el Trabajo, Madrid, España \\ ${ }^{3}$ Servicio de Prevención de SSIBE_Fundación Hospital de Palamós, Palamós (Girona). España \\ ${ }^{4}$ Departamento de Ciencias Médicas, Facultad de Medicina, Universitat de Girona, Girona. España
}

Información · Information

Este documento es resultado de las actividades de la Sección de Medicina del Trabajo de la UEMS (Unión Europea de Médicos Especialistas: www.uems.net)

Fechas · Dates

Recibido: 2019.12.27

Publicado: 2020.01.15

\section{Correspondencia $\cdot$ Corresponding Author}

Mari Cruz Rodríguez-Jareño

mcrodriguezj@gmail.com 
El pasado 23 de noviembre se celebró en Lisboa el segundo congreso internacional de la Sociedad Portuguesa de Medicina del Trabajo (SPMT), a continuación de su décimoquinto congreso nacional que tuvo lugar el 21 y 22.

Uno de los objetivos planteados por su presidente es el de estrechar lazos con otras Sociedades de Medicina del Trabajo y en concreto entre Sociedades vecinas de la península ibérica, así como con diferentes organismos internacionales.

Tras la bienvenida dada por su presidente, Dr Jorge Barroso Dias, se inició el congreso internacional con una presentación por video del presidente de ACOEM (American College of Occupational and Environmental Medicine). El hilo conductor del congreso giraba alrededor de las aportaciones de la Medicina del Trabajo a la Salud Pública, para resaltar el reciente reconocimiento de la Sociedad Portuguesa de Medicina del Trabajo como entidad de utilidad pública en Portugal.

La jornada se enfocó principalmente a presentar las actividades y contribución de diferentes organismos internacionales, y se contó con las intervenciones de Joaquim Pintado Nunes de la Organización Internacional del Trabajo (OIT), de Claudina Nogueira de la Comisión Internacional de Salud Laboral $(\mathrm{ICOH})$ y de Alenka Skerjanc, presidenta de la sección de Medicina del Trabajo de la Unión Europea de Médicos Especialistas (UEMS).

Durante la mesa de la mañana, el representante portugués (Pedro Gustavo Reis) y la representante española (Mari Cruz Rodríguez-Jareño) de la UEMS explicaron las aportaciones de sus respectivos países a la sección de Medicina del Trabajo de la UEMS.

En la sesión de la tarde intervinieron Alfonso Apellaniz, presidente de la SEMST (Sociedad Española de Medicina y Seguridad en el Trabajo), con una presentación sobre la situación actual de las Enfermedades Profesionales en España, y Juan Luis Cabanillas, miembro de la junta de la AEEMT (Asociación Española de Especialistas en Medicina del Trabajo), con una presentación sobre el programa andaluz de Promoción de la Salud en el Trabajo, así como Mari Cruz Rodríguez miembro de la junta de la SCSL (Societat Catalana de Salut Laboral) que expuso el proyecto Europeo EWA sobre alcohol y otras drogas en el trabajo, y la guía y el programa «A la feina alcohol 0.0» desarrollados por la Generalitat de Cataluña.

Se puede acceder al programa del congreso nacional e internacional a través de la página web de la Sociedad Portuguesa de Medicina del Trabajo o en el siguiente enlace: https://595f1784-567a-4ebc-8f5e-874332227bba.filesusr.com/ugd/ a7d6ed_0bf89ab9f1a94bbfb9b0cfa636fada64.pdf

SPMT (Sociedad Portuguesa de Medicina del Trabajo) https://www.spmtrabalho. org/

ICOH (International Commission on Occupational Health) http://www.icohweb. org/site/homepage.asp 
UEMS (Unión Europea de Médicos Especialistas) https://uems-occupationalmedicine.org/

ACOEM (American College of Occupational and Environmental Medicine) https:// acoem.org/

SEMST (Sociedad Española de Medicina y Seguridad en el Trabajo) https://www. semst.org/

AEEMT (Asociación Española de Especialistas en Medicina del Trabajo) http:// www.aeemt.com/web/

SCSL (Societat Catalana de Salut Laboral) http://www.salutlaboral.com/

\section{Segon congrés internacional de la Societat Portuguesa de Medicina del Treball}

El passat 23 de novembre es va celebrar a Lisboa el segon congrés internacional de la Societat Portuguesa de Medicina del Treball (SPMT), a continuació del seu quinzè congrés nacional que va tenir lloc el 21 i 22.

Un dels objectius plantejats pel seu president és el d'estrènyer llaços amb altres Societats de Medicina del Treball i en concret entre societats veïnes de la península ibèrica, així com amb diferents organismes internacionals.

Després de la benvinguda donada pel seu president, Dr Jorge Barroso Dias, es va iniciar el congrés internacional amb una presentació per vídeo del president d'ACOEM (American College of Occupational and Environmental Medicine). El fil conductor del congrés girava al voltant de les aportacions de la Medicina del Treball a la Salut Pública, per ressaltar el recent reconeixement de la Societat Portuguesa de Medicina del Treball com a entitat d'utilitat pública a Portugal.

La jornada es va enfocar principalment a presentar les activitats i contribució de diferents organismes internacionals, i es va comptar amb les intervencions de Joaquim Pintado Nunes de l'Organització Internacional del Treball (OIT), de Claudina Nogueira de la Comissió Internacional de Salut Laboral (ICOH) i de Alenka Skerjanc, presidenta de la secció de Medicina del Treball de la Unió Europea de Metges Especialistes (UEMS).

Durant la taula del matí, el representant portuguès (Pedro Gustavo Reis) i la representant espanyola (Mari Cruz Rodríguez-Jareño) de la UEMS van explicar les aportacions dels seus respectius països a la secció de Medicina del Treball de la UEMS.

A la sessió de la tarda van intervenir Alfonso Apellaniz, president de la SEMST (Sociedad Española de Medicina i Seguridad en el Trabajo), amb una presentació sobre la situació actual de les Malalties Professionals a Espanya, i Juan Luis Ca- 
banillas, membre de la junta de la AEEMT (Asociación Española de Especialistas en Medicina del Trabajo), amb una presentació sobre el programa andalús de Promoció de la Salut en el Treball, així com Mari Cruz Rodríguez membre de la junta de la SCSL (Societat Catalana de Salut Laboral) que va exposar el projecte Europeu EWA sobre alcohol i altres drogues a la feina, i la guia i el programa «a la feina alcohol 0.0» desenvolupats per la Generalitat de Catalunya.

Es pot accedir a el programa del congrés nacional i internacional a través de la pàgina web de la Societat Portuguesa de Medicina del Treball o en el següent enIlaç: https://595f1784-567a-4ebc-8f5e-874332227bba.filesusr.com/ugd/a7d6ed_0bf89ab9f1a94bbfb9b0cfa636fada64.pdf

SPMT (Sociedad Portuguesa de Medicina del Trabajo) https://www.spmtrabalho. org/

ICOH (International Commission on Occupational Health) http://www.icohweb. org/site/homepage.asp

UEMS (Unión Europea de Médicos Especialistas) https://uems-occupationalmedicine.org/

ACOEM (American College of Occupational and Environmental Medicine) https:// acoem.org/

SEMST (Sociedad Española de Medicina y Seguridad en el Trabajo) https://www. semst.org/

AEEMT (Asociación Española de Especialistas en Medicina del Trabajo) http:// www.aeemt.com/web/

SCSL (Societat Catalana de Salut Laboral) http://www.salutlaboral.com/ 